\title{
II modello degli snodi decisionali nella cura del tabagismo: uno studio osservazionale
}

\section{The decision-making model in the treatment of tobacco addiction: an observational study}

\author{
Franco Badii, Sarah Grossi
}

\section{Riassunto}

Introduzione: La sensazione di automatismo gioca un ruolo fondamentale nei fenomeni di dipendenza. Pertanto, nel processo terapeutico, diventa importante intervenire per aumentare la consapevolezza del paziente riguardo alle proprie azioni. Per fare questo abbiamo sviluppato il modello degli "snodi decisionali". Scopo del presente lavoro è la valutazione dell'impatto, al momento del primo contatto con l'ambulatorio per il tabagismo del Ser.D., di un intervento mirato agli snodi decisionali in termini di consapevolezza e di possibile ricaduta sulla dipendenza da tabacco.

Metodi: Sono stati presi in considerazione 78 pazienti. A un gruppo (39) è stata applicata esclusivamente la procedura standard; al secondo gruppo (39) è stata aggiunta una spiegazione del modello "snodi decisionali". In entrambi i casi, sono stati misurati i livelli di monossido di carbonio (CO) e valutato il numero di sigarette (self-report), in occasione della prima e seconda visita.

Risultati: I dati sembrano sostenere l'importanza della consapevolezza e della percezione della agency nel contrastare il mantenimento della dipendenza. Si è infatti osservata una riduzione sia del $\mathrm{CO}$ che del numero di sigarette fumate maggiore nel gruppo decisionale rispetto a quello standard. Applicando il test t di Student alle variazioni di CO risulta che la differenza tra le medie osservate è significativa e non casuale per $p<0,01 ; t=3,2501$; gradi di libertà $=76$; $p$ (livello di significatività): 0,0017 . Lo stesso test non evidenzia significatività a meno del $5 \%$ in relazione al numero di sigarette nei due gruppi.

Conclusioni: La possibilità di agire sulla dipendenza da tabacco già al primo colloquio ha ricadute positive, oltre che sulle condizioni del paziente, sulla percezione della propria autoefficacia e può portare a una maggior attivazione nel corso del proseguimento del lavoro terapeutico. È opportuno tenere in considerazione l'aspetto dei drop-out precoci, per cui è indicato ottimizzare il messaggio espresso in occasione della prima visita.

Parole chiave: Dipendenza, agency, decisione.

\section{Abstract}

Introduction: The automatic reaction plays a fundamental role in the addiction phenomena. Thus, it is important to intervene in the therapeutic process in order to increase the patient's awareness of his own actions. To do that we have developed the decision making-model. The aim of the present paper is the evaluation of the impact - at the moment of the first contact with Smoking Cessation Centre - on an intervention aiming at decision-making steps and options, considering awareness and eventual positive effect on tobacco addiction.

Methods: 78 patients were considered. One group (39) underwent just the standard procedure. The second group (39) was also given an explanation of the decision - making steps and options. In both cases the carbon monoxide (CO) levels were measured and the number of cigarettes assessed (self-report) at the first and second visits.

Results: The data seem to support the importance of the awareness and of the perception of agency in contrasting the persistence of addiction. The application of the Student's t test to the CO variations, 
prove that the difference between the observed data is significant and not random for $p<0.01$; $t=3.2501$; degrees of freedom $=76 ; p$ (significance level): 0.0017 . The same test does not show significance at less than $5 \%$ in relation to the number of cigarettes in the two groups.

Conclusions: The possibility of acting on tobacco addiction already from the first interview has positive effects both on the condition of the patient and on the perception of his efficacy and can lead to a greater responsibility and freedom of choice during the continuation of the therapy

Keywords: Addiction, agency, choice.

\section{Introduzione}

Una delle caratteristiche dei quadri clinici di dipendenza è costituita dalla perdita di controllo, correlata a processi neurobiologici che si manifestano non solo in relazione al piacere, ma anche in relazione all'importanza che il comportamento di dipendenza assume dal punto di vista individuale, e che si manifesta con modalità connesse ad aspetti situazionali e di contesto. Tali affermazioni fanno riferimento alla teoria della salienza incentiva di Robinson e Berridge [1-8] e quindi al ruolo della dopamina nell'attivare i processi che caratterizzano la dipendenza. $\mathrm{Ci}$ è parso interessante, in particolare, il ruolo svolto da questo neurotrasmettitore nel determinare il passaggio da un comportamento controllato da aspettative esplicite, del tipo act-outcome, a un comportamento del tipo stimolo-risposta, dando origine a una situazione di abitudine [9] percepita soggettivamente come automatismo [10].

Partendo da una formazione psicoterapica in Analisi Transazionale che ha come cardine il ruolo attribuito alla decisione individuale $[11,12]$, abbiamo costruito un modello ("snodi decisionali") che affronta il senso di automatismo, anche facendo riferimento alle teorie che, nelle dipendenze, mettono in risalto la responsabilità individuale, non intesa in senso morale, ma nel senso di agency [13-16].

Le azioni correlate alla dipendenza si possono infatti dividere in due parti, una sotto il controllo dell'individuo, seppur influenzato dal craving, l'altra che una volta attivata, diventa irreversibile [10]. Ovviamen- te maggiore è la distanza dall'area dell'irreversibilità, maggiore è il controllo che il soggetto riesce a esercitare sul proprio comportamento, anche in presenza di craving. In questo senso, per un fumatore, è più facile decidere di non portare con sé il pacchetto di sigarette, che cercare di fronteggiare il craving avendo il pacchetto in tasca, ed è quasi impossibile non accendere la sigaretta una volta che la si ha in mano. Questo corrisponde all'attivazione del comportamento irreversibile che procede seguendo un proprio ciclo caratteristico, giungendo a conclusione con la fine dell'atto (accendere la sigaretta). Solitamente il paziente affetto da dipendenza ritiene di dover esercitare il controllo in prossimità della linea di irreversibilità, quando ciò è estremamente difficile.

In definitiva, le azioni attuate dal soggetto dipendente sono volontarie, in quanto determinate da atti intenzionali, ma avvengono senza la relativa consapevolezza, in quanto inserite in un meccanismo percepito come automatico, basato su un'abitudine. La percezione di questa abitudine ha una intensità che aumenta progressivamente con l'avvicinarsi dei comportamenti alle situazioni che caratterizzano l'inizio del processo irreversibile.

A questo punto si innesca il circuito della dipendenza che nel nostro modello comprende tre aspetti salienti: automatismo, distorsioni cognitive e ripetizione [10].

\section{Automatismo}

È la sensazione del soggetto di essere in balia della dipendenza.

La possibilità di pensare e agire di- versamente scompare e permane il comportamento legato alla dipendenza.

Le azioni correlate alla dipendenza sono vissute come inevitabili e ineluttabili.

\section{Distorsioni cognitive}

Nell'evoluzione della dipendenza si sviluppa una logica finalizzata al suo mantenimento [17]. Tale logica si manifesta quando la dipendenza potrebbe essere messa in discussione da elementi provenienti sia da fonti esterne che interne all'individuo.

Le distorsioni cognitive si possono manifestare come:

- alibi o scuse che il soggetto accampa per affermare anche a se stesso l'impossibilità di agire diversamente, sostenendo la propria passività rispetto alla dipendenza;

- pensieri caratterizzati da contenuti in apparenza formalmente congrui, spesso diffusi anche a livello culturale, ma in realtà basati su aspetti pregiudiziali, del tutto infondati, o incongrui rispetto al contesto in cui vengono applicati;

- convinzioni e aspettative irrealistiche.

\section{Ripetizione}

È la conseguenza della sensazione di automatismo. Se manca la consapevolezza delle decisioni che si prendono, è naturale percepire unicamente la ripetizione di un'azione, rinforzando così la sensazione di impotenza.

Nella fase in cui il processo è ancora reversibile, sono individuabili dei precisi momenti in cui una sequenza di decisioni può condurre 
a due diverse opzioni, una sorta di "bivio": comportamento di dipendenza oppure un comportamento diverso. Abbiamo definito questi momenti "snodi decisionali".

L'abitudine fa sì che sia privilegiata la scelta legata al comportamento relativo alla dipendenza, cosicché il soggetto ha la percezione che lo stimolo segua immediatamente l'azione, senza consapevolezza delle varie decisioni che, a partire dallo stimolo, conducono all'azione stessa. Per esempio, l'atto di accendere una sigaretta è vissuto da un fumatore come un tutt'uno, un vero e prorio automatismo, ma in realtà è composto da una serie di atti (prendere in mano il pacchetto, estrarre una sigaretta, prendere I'accendino, avvicinare la sigaretta alla fiamma, aspirare), ognuno dei quali dovuto a una decisione presa dal soggetto.

Pertanto, nell'ambito di un processo terapeutico diventa importante stimolare e ampliare la consapevolezza dei processi mentali che dallo stimolo portano all'azione, per recuperare la situazione iniziale, di comportamento act-outcome. II paziente deve essere aiutato a riconoscere i momenti in cui prende decisioni (snodi decisionali), facendo emergere il ruolo della responsabilità soggettiva, nei termini di agency, e rinforzando positivamente anche minimi cambiamenti.

Da un punto di vista pratico, nel corso del colloquio clinico si presta attenzione all'emergere di riferimenti all'automatismo: spesso il paziente tabagista descrive le sue abitubini correlate al fumo con frasi del tipo "capita", "mi succede che...", espressioni inevitabili, vista la centralità di questa sensazione nel vissuto della persona dipendente.

Si evidenzia al paziente la sua tendenza a spiegare in termini passivi la propria esperienza, mettendo piuttosto in rilievo il suo ruolo come protagonista delle proprie scelte. $\mathrm{Si}$ possono utilizzare, a tal fine, frasi del tipo: "lei è abituato dalla sua di- pendenza a pensare così, ma se ci riflette è lei che fa le cose, non capitano"; "è lei che prende in mano sigaretta e accendino e attua una serie di gesti che portano ad accenderla e a inalare il fumo, ma dopo tanti anni che ripete questa sequenza tante volte al giorno è possibile che non ci faccia più caso".

Si cerca di rinforzare la sensazione di possibilità di azione che si vuole indurre nel paziente utilizzando esempi e/o metafore: "I'abitudine è come un'autostrada a sei corsie, che lei percorre a grande velocità; le possibilità alternative sono dei piccoli bivi mal segnalati davanti ai quali è abituato a sfrecciare senza vederli". Molto spesso l'intervento sulla sensazione di automatismo attiva le dissonanze cognitive: il soggetto ricorre ad argomenti a sostegno della percezione di passività nel suo comportamento ("io sono una persona debole", "è una questione di forza di volontà"). Anche queste affermazioni vanno riprese e decodificate nell'ottica della possibilità di azione ("è sicuro che in realtà non si tratti di poca abitudine a far diversamente?"; "si tratta di forza di volontà o di non aver chiaro cosa fare e, soprattutto, quando farlo?").

Nel corso del colloquio è opportuno anche far riferimento ad aspetti teorici, come la linea di irreversibilità, la possibilità di controllo in base alla lontananza da essa. II clima del colloquio deve essere non giudicante, I'approccio empatico, le osservazioni fatte dal terapeuta devono apparire come semplici informazioni, stimoli in grado di attivare la riflessione del paziente. L'obiettivo esplicitato non consiste nel cambiare il comportamento, ma nel focalizzare i momenti (snodi) in cui vengono prese le decisioni, relativamente al comportamento di dipendenza. Questo ha lo scopo di non gravare il soggetto di eccessive aspettative e di entrare in empatia con lui mostrando comprensione per le sue difficoltà ad affrontare un cambiamento. In effetti, si è osservato che il report del paziente al colloquio successivo faceva spesso riferimento anche a un cambiamento.

La letteratura scientifica ha evidenziato l'efficacia degli interventi di motivational interviewing a sessione singola nell'ambito della dipendenza $[18,19]$. Dagli studi emerge che una singola sessione può fungere da catalizzatore nei processi legati al cambiamento, sia relativamente alla consapevolezza della possibilità di cambiare sia rispetto al prepararsi al cambiamento o prendere la decisione di cambiare [18].

Sulla base di queste osservazioni, abbiamo inserito nella pratica dell'ambulatorio per il tabagismo un intervento sugli snodi decisionali durante il primo colloquio, integrando il colloquio motivazionale standard, anche per cercare di sfruttare la motivazione del paziente, che si suppone alta nel momento in cui, autonomamente, si rivolge per la prima volta a un servizio.

Si sono utilizzati anche alcuni aspetti che caratterizzano la terapia a seduta singola [20], che ha come presupposto il ruolo centrale e attivo svolto dal soggetto. Altrettanto importante, nel mindset dell'operatore, è la convinzione che la soluzione di grandi problemi non richieda grandi cambiamenti [20]. È infatti fondamentale, per garantire l'efficacia dell'intervento, che, nel corso del colloquio, l'operatore mantenga fermo il convincimento che sia possibile, modificando semplici abitudini, raggiungere un complesso cambiamento.

Dopo qualche mese dall'introduzione di questo approccio nell'attività dell'ambulatorio, abbiamo avuto conferma della sensazione che il primo colloquio svolto con queste modalità avesse un impatto positivo sul paziente, per cui abbiamo confrontato i parametri in nostro possesso, relativi ai pazienti seguiti con l'approccio decisionale [24], con i dati di pazienti ai quali in passato era stato proposto il trattamento standard con elementi di colloquio motivazionale [21-23]. 
La Tabella 1 riporta le caratteristiche dell'intervento standard e di quello decisionale.

\section{Materiali e metodi}

Il campione oggetto dello studio è costituito da 78 pazienti afferenti all'ambulatorio tabagismo della ASL2 savonese dal giorno 8 aprile al 9 dicembre 2019 tutti affetti da disturbo da uso di tabacco di grado grave secondo il DSM-5 [25]. II numero complessivo è stato di 40 uomini e 38 donne, con età media di 52 anni. È stato utilizzato il collo- quio decisionale con i 39 tabagisti che si sono presentati per intraprendere un percorso ambulatoriale dal 12 agosto al 9 dicembre 2019; abbiamo poi esaminato un egual numero di pazienti che si erano rivolti all'ambulatorio nei quattro mesi precedenti e che avevano seguito il trattamento standard. Abbiamo, per maggior chiarezza, definito i due gruppi rispettivamente decisionale e standard.

La Tabella 2 riporta la distribuzione dei due gruppi per sesso, età media, grado di dipendenza, livello di motivazione, numero di siga-

Tabella 1 Caratteristiche del colloquio standard e di quello decisionale.

\begin{tabular}{ll} 
Colloquio standard & Colloquio decisionale \\
Approccio empatico & Approccio empatico \\
\hline Atteggiamento non giudicante & Atteggiamento non giudicante \\
\hline $\begin{array}{l}\text { Ragionamento sulle abitudini } \\
\text { fumo-correlate }\end{array}$ & Ragionamento sulle abitudini fumo-correlate \\
\hline Aumentare la frattura interiore & $\begin{array}{l}\text { Affrontare la sensazione di automatismo } \\
\text { e le distorsioni cognitive }\end{array}$ \\
\hline $\begin{array}{l}\text { Accogliere e utilizzare } \\
\text { le resistenze }\end{array}$ & $\begin{array}{l}\text { Aumentare la consapevolezza degli snodi } \\
\text { decisionali }\end{array}$ \\
\hline Sostenere l'autoefficacia & $\begin{array}{l}\text { Enfatizzare il ruolo centrale svolto dal soggetto } \\
\text { nel processo di cambiamento (agency) }\end{array}$ \\
\hline
\end{tabular}

Tabella 2 Distribuzione per sesso, età media, grado di dipendenza, livello di motivazione, numero di sigarette/die e valore di CO nell'espirato.

\begin{tabular}{|c|c|c|}
\hline & Gruppo standard & Gruppo decisionale \\
\hline Sesso & 21 uomini, 18 donne & 19 uomini, 20 donne \\
\hline Età media & 51,6 aа & 52,3 aа \\
\hline Fagerström & $\begin{array}{l}\text { Dipendenza lieve (0-2): } 1 \\
\text { Dipendenza media (3-4): } 6 \\
\text { Dipendenza forte (5-6): } 14 \\
\text { Dipendenza molto forte (7-10): } 18\end{array}$ & $\begin{array}{l}\text { Dipendenza lieve (0-2): } 2 \\
\text { Dipendenza media (3-4): } 11 \\
\text { Dipendenza forte (5-6): } 9 \\
\text { Dipendenza molto forte (7-10): } 17\end{array}$ \\
\hline Q-MAT & $\begin{array}{l}\text { Motivazione insufficiente }(\leq 6): 1 \\
\text { Motivazione media }(7-12): 9 \\
\text { Motivazione buona }(\geq 13): 29\end{array}$ & $\begin{array}{l}\text { Motivazione insufficiente }(\leq 6): 2 \\
\text { Motivazione media }(7-12): 6 \\
\text { Motivazione buona }(\geq 13): 31\end{array}$ \\
\hline N. sig/die & $\begin{array}{l}\leq 10 \text { sig/die: } 3 \\
\text { tra } 11 \text { e } 20 \text { sig/die: } 20 \\
>20 \text { sig/die: } 16\end{array}$ & $\begin{array}{l}\leq 10 \text { sig/die: } 7 \\
\text { tra } 11 \text { e } 20 \text { sig/die: } 17 \\
>20 \text { sig/die: } 15\end{array}$ \\
\hline $\mathrm{CO}$ (ppm) & $\begin{array}{l}\text { 3-10: } 8 \\
\text { 11-20: } 13 \\
21-50: 18\end{array}$ & $\begin{array}{l}3-10: 3 \\
11-20: 13 \\
21-50: 23\end{array}$ \\
\hline
\end{tabular}

rette/die e valore di monossido di carbonio (CO) nell'espirato (ppm). Le Figure 1A e 1B mostrano la gravità di dipendenza in base al punteggio ottenuto con il test di Fagerström rispettivamente nei gruppi decisionale e standard.

In questo studio osservazionale è stata impiegata per entrambi i gruppi di pazienti la medesima procedura che può essere così schematizzata:

- In occasione della prima visita, si è raccolta l'anamnesi tabaccologica (N. sig/die, età d'esordio, pregressi tentativi di disassuefazione), è stato eseguito un colloquio (decisionale o standard a seconda del gruppo), sono stati proposti il test per la nicotino-dipendenza di Fagerström [26-28] e il test motivazionale Q-MAT [29], si è consegnato il diario del fumatore con l'indicazione di registrare, contestualmente all'atto di fumare, tutte le sigarette fumate ogni giorno, associando l'invito a ridurne il numero; infine è stato misurato il $\mathrm{CO}$ nell'espirato, mediante un dispositivo portatile [3034].

- Nel corso della seconda visita, tutti e 78 i pazienti sono stati sottoposti nuovamente alla misurazione del $\mathrm{CO}$ e sono stati esaminati i diari da loro compilati. Risulta pertanto evidente che l'unica differenza di approccio per i due gruppi è stata l'integrazione del colloquio con il modello incentrato sugli "snodi decisionali", rivolta esclusivamente nel corso della prima visita, al gruppo decisionale.

Le Tabelle $\mathbf{3}$ e $\mathbf{4}$ mostrano rispettivamente i valori di $\mathrm{CO}$ e numero di sigarette giornaliere dei due gruppi, al baseline e alla seconda visita. Si precisa che lo stesso medico ha effettuato tutte le visite e i colloqui ai tabagisti oggetto dello studio; per tutte le misurazioni di $\mathrm{CO}$ nell'espirato è stato utilizzato lo stesso dispositivo portatile munito di 


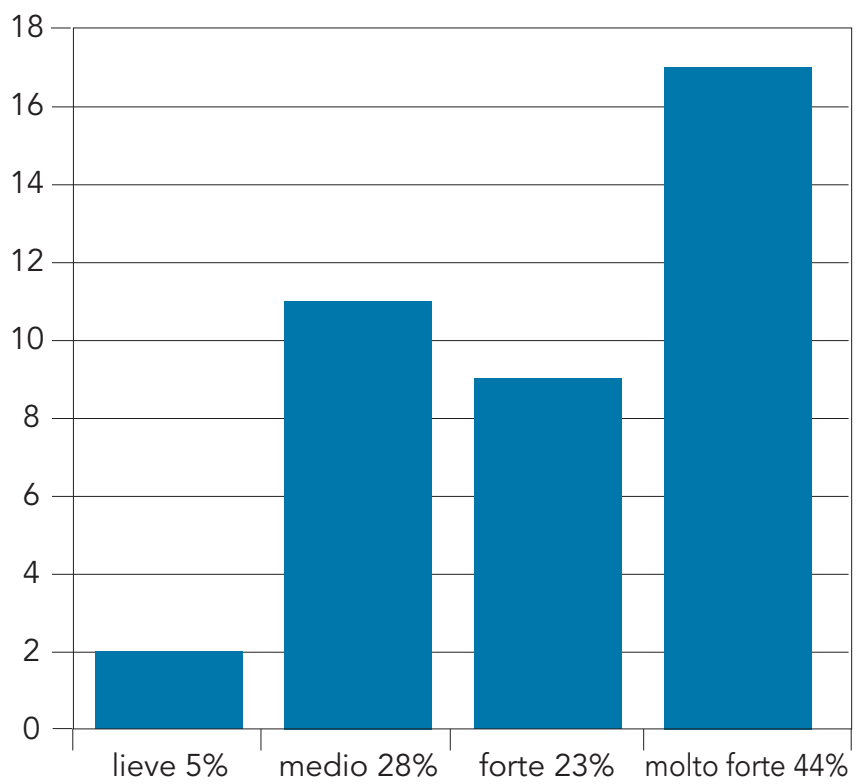

Figura 1A Gravità della dipendenza in base al test di Fagerström nel gruppo decisionale.

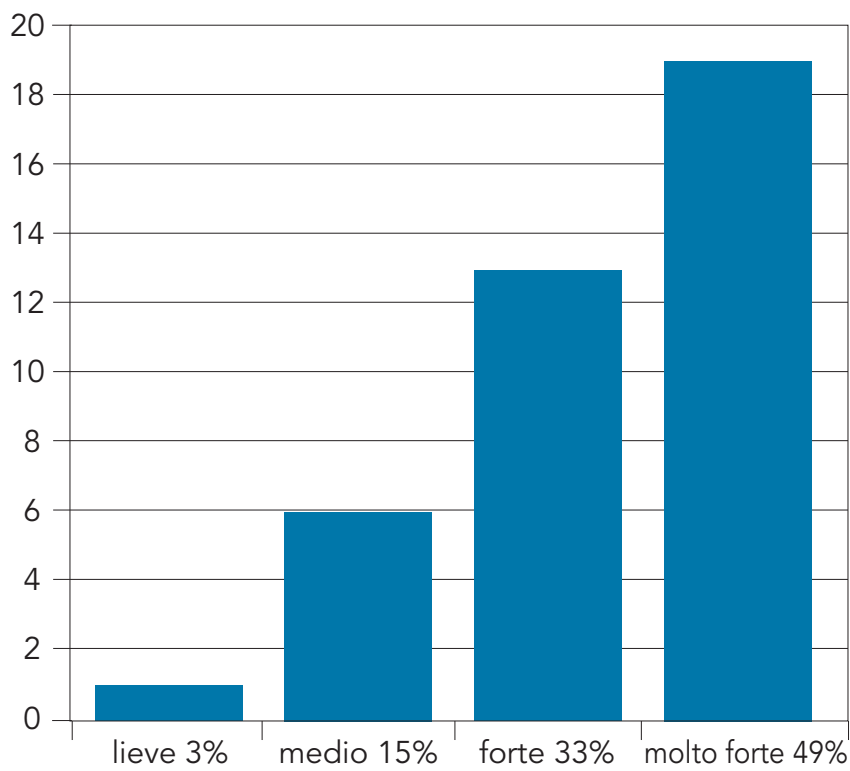

Figura 1B Gravità della dipendenza in base al test di Fagerström nel gruppo standard.

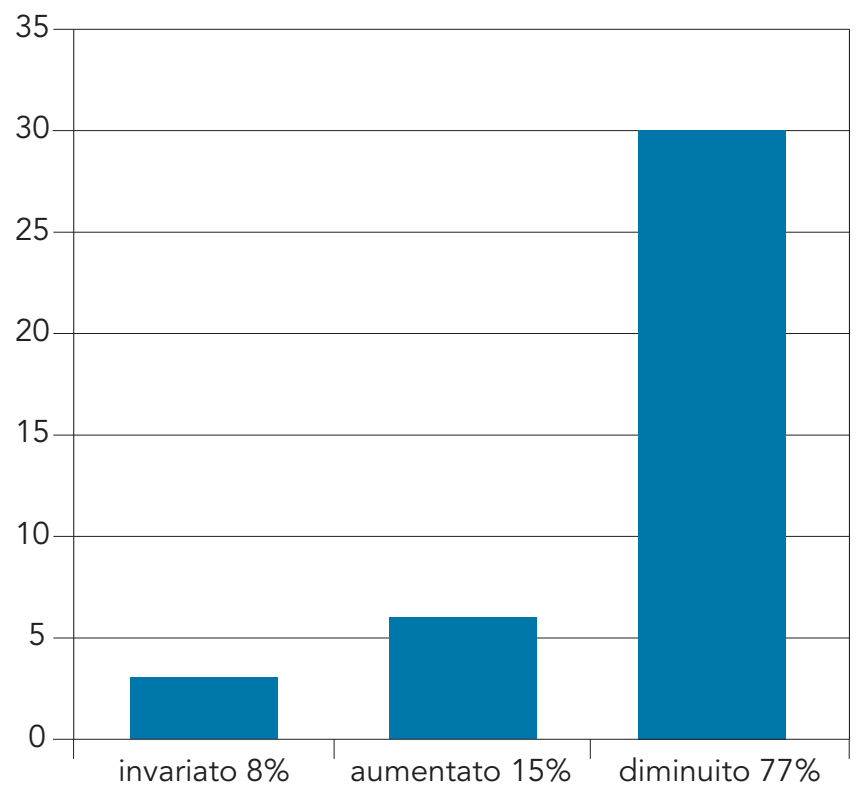

Figura 2A Variazione del livello di CO nel gruppo decisionale.

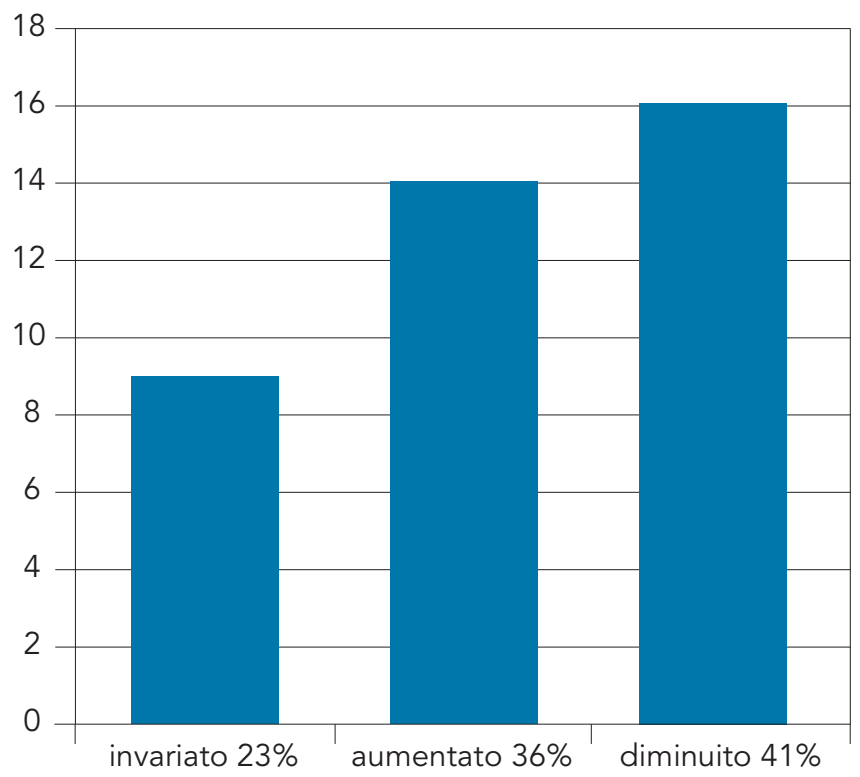

Figura 2B Variazione del livello di CO nel gruppo standard. appositi boccagli monouso; la durata dei colloqui è stata pressoché invariata in entrambi i gruppi (45 minuti per quanto riguarda le prime visite, 30-35 minuti per le seconde visite); il periodo intercorso tra la prima e la seconda visita è stato di 14 giorni. In nessun caso nella prima visita sono stati prescritti farmaci per disassuefazione da nicotina.
Si è presa in considerazione la variazione dei parametri rilevati dopo il primo colloquio, non avendo ovviamente come target la cessazione tabagica, ma il loro scostamento in termini di riduzione del fumo. Si è pertanto deciso di confrontare le variazioni dei livelli di $\mathrm{CO}$ e del numero di sigarette tra prima e seconda visita in entrambi i gruppi. Le differenze riscontrate sono ripor- tate, accanto ai rispettivi punteggi ottenuti al test di Fagerström e Q-MAT, nelle Tabelle 5 e 6.

I grafici a istogrammi delle Figure 2A e 2B mostrano l'andamento del $\mathrm{CO}$, rispettivamente nel gruppo decisionale e standard. È evidente che il numero di pazienti che ha presentato una riduzione del livello di $\mathrm{CO}$, in occasione della seconda visita, è nettamente superiore nel 
Tabella 3 Gruppo decisionale.

\begin{tabular}{|c|c|c|c|}
\hline $\begin{array}{c}\mathrm{CO} \\
1^{\circ} \text { colloquio }\end{array}$ & $\begin{array}{c}\mathrm{CO} \\
2^{\circ} \text { colloquio }\end{array}$ & $\begin{array}{l}\text { N. sigarette } \\
1^{\circ} \text { colloquio }\end{array}$ & $\begin{array}{l}\text { N. sigarette } \\
2^{\circ} \text { colloquic }\end{array}$ \\
\hline 14 & 10 & 10 & 5 \\
\hline 28 & 10 & 40 & 2 \\
\hline 28 & 28 & 30 & 20 \\
\hline 13 & 9 & 15 & 7 \\
\hline 10 & 0 & 4 & 0 \\
\hline 14 & 0 & 18 & 0 \\
\hline 27 & 16 & 30 & 18 \\
\hline 28 & 10 & 13 & 5 \\
\hline 22 & 16 & 25 & 15 \\
\hline 28 & 9 & 43 & 10 \\
\hline 31 & 32 & 20 & 15 \\
\hline 8 & 23 & 15 & 12 \\
\hline 20 & 24 & 10 & 12 \\
\hline 24 & 20 & 25 & 10 \\
\hline 23 & 5 & 10 & 0 \\
\hline 22 & 18 & 30 & 12 \\
\hline 18 & 10 & 12 & 6 \\
\hline 18 & 12 & 18 & 15 \\
\hline 33 & 33 & 35 & 35 \\
\hline 33 & 36 & 20 & 10 \\
\hline 31 & 20 & 20 & 12 \\
\hline 23 & 18 & 16 & 10 \\
\hline 30 & 0 & 23 & 0 \\
\hline 31 & 25 & 20 & 16 \\
\hline 20 & 8 & 12 & 6 \\
\hline 20 & 15 & 30 & 20 \\
\hline 18 & 14 & 20 & 15 \\
\hline 28 & 20 & 25 & 18 \\
\hline 29 & 25 & 25 & 20 \\
\hline 29 & 16 & 20 & 15 \\
\hline 7 & 18 & 3 & 18 \\
\hline 16 & 12 & 13 & 7 \\
\hline 13 & 13 & 10 & 8 \\
\hline 18 & 11 & 11 & 6 \\
\hline 27 & 22 & 15 & 11 \\
\hline 18 & 20 & 9 & 12 \\
\hline 28 & 22 & 40 & 30 \\
\hline 32 & 32 & 35 & 30 \\
\hline 34 & 30 & 25 & 13 \\
\hline
\end{tabular}

Tabella 4 Gruppo standard.

\begin{tabular}{|c|c|c|c|}
\hline $\begin{array}{c}\mathrm{CO} \\
1^{\circ} \text { colloquio }\end{array}$ & $\begin{array}{c}\mathrm{CO} \\
2^{\circ} \text { colloquio }\end{array}$ & $\begin{array}{l}\text { N. sigarette } \\
1^{\circ} \text { colloquio }\end{array}$ & $\begin{array}{l}\text { N. sigarette } \\
2^{\circ} \text { colloquio }\end{array}$ \\
\hline 23 & 34 & 10 & 15 \\
\hline 27 & 20 & 15 & 10 \\
\hline 3 & 15 & 3 & 7 \\
\hline 12 & 8 & 13 & 13 \\
\hline 23 & 20 & 33 & 19 \\
\hline 9 & 14 & 18 & 15 \\
\hline 24 & 18 & 15 & 13 \\
\hline 28 & 28 & 20 & 12 \\
\hline 31 & 31 & 20 & 14 \\
\hline 31 & 23 & 30 & 15 \\
\hline 27 & 27 & 30 & 30 \\
\hline 24 & 14 & 13 & 10 \\
\hline 21 & 10 & 15 & 12 \\
\hline 16 & 15 & 20 & 13 \\
\hline 29 & 30 & 20 & 13 \\
\hline 31 & 24 & 40 & 40 \\
\hline 34 & 29 & 20 & 13 \\
\hline 11 & 7 & 16 & 7 \\
\hline 26 & 35 & 37 & 27 \\
\hline 11 & 23 & 27 & 15 \\
\hline 7 & 15 & 18 & 15 \\
\hline 14 & 28 & 30 & 15 \\
\hline 12 & 23 & 20 & 20 \\
\hline 29 & 29 & 50 & 23 \\
\hline 15 & 15 & 40 & 40 \\
\hline 21 & 14 & 20 & 15 \\
\hline 9 & 1 & 15 & 1 \\
\hline 25 & 15 & 45 & 20 \\
\hline 19 & 7 & 27 & 20 \\
\hline 11 & 11 & 5 & 6 \\
\hline 15 & 38 & 20 & 20 \\
\hline 10 & 18 & 20 & 20 \\
\hline 10 & 12 & 35 & 32 \\
\hline 14 & 18 & 20 & 20 \\
\hline 7 & 7 & 22 & 18 \\
\hline 11 & 11 & 23 & 15 \\
\hline 16 & 16 & 20 & 10 \\
\hline 35 & 22 & 22 & 20 \\
\hline 4 & 7 & 25 & 22 \\
\hline
\end{tabular}


Tabella 5 Gruppo decisionale.

\begin{tabular}{|c|c|c|c|c|c|c|c|c|c|}
\hline Paz & Fagerström & Q-MAT & Sesso & $\mathrm{CO} 1^{\circ} \mathrm{coll}$. & $\mathrm{CO} 2^{\circ}$ coll. & $\begin{array}{l}\text { N. sigarette } \\
1^{\circ} \text { colloquio }\end{array}$ & $\begin{array}{l}\text { N. sigarette } \\
2^{\circ} \text { colloquio }\end{array}$ & $\begin{array}{c}\text { Variazione } \\
\text { CO }\end{array}$ & $\begin{array}{l}\text { Variazione } \\
\text { N. sigarette }\end{array}$ \\
\hline 1 & 6 & 19 & $\mathrm{~F}$ & 14 & 10 & 10 & 5 & -4 & -5 \\
\hline 2 & 7 & 20 & $M$ & 28 & 10 & 40 & 2 & -18 & -38 \\
\hline 3 & 7 & 20 & $\mathrm{~F}$ & 28 & 28 & 30 & 20 & 0 & -10 \\
\hline 4 & 4 & 14 & $\mathrm{~F}$ & 13 & 9 & 15 & 7 & -4 & -8 \\
\hline 5 & 4 & 15 & $M$ & 10 & 0 & 4 & 0 & -10 & -4 \\
\hline 6 & 3 & 20 & $M$ & 14 & 0 & 18 & 0 & -14 & -18 \\
\hline 7 & 9 & 18 & $M$ & 27 & 16 & 30 & 18 & -11 & -12 \\
\hline 8 & 8 & 8 & $\mathrm{~F}$ & 28 & 10 & 13 & 5 & -18 & -8 \\
\hline 9 & 7 & 17 & $M$ & 22 & 16 & 25 & 15 & -6 & -10 \\
\hline 10 & 10 & 20 & $\mathrm{M}$ & 28 & 9 & 43 & 10 & -19 & -33 \\
\hline 11 & 7 & 16 & $\mathrm{~F}$ & 31 & 32 & 20 & 15 & 1 & -5 \\
\hline 12 & 5 & 9 & $\mathrm{~F}$ & 8 & 23 & 15 & 12 & 15 & -3 \\
\hline 13 & 4 & 14 & M & 20 & 24 & 10 & 12 & 4 & 2 \\
\hline 14 & 4 & 12 & $\mathrm{~F}$ & 24 & 20 & 25 & 10 & -4 & -15 \\
\hline 15 & 4 & 19 & M & 23 & 5 & 10 & 0 & -18 & -10 \\
\hline 16 & 2 & 20 & $\mathrm{M}$ & 22 & 18 & 30 & 12 & -4 & -18 \\
\hline 17 & 4 & 13 & $\mathrm{~F}$ & 18 & 10 & 12 & 6 & -8 & -6 \\
\hline 18 & 5 & 17 & $M$ & 18 & 12 & 18 & 15 & -6 & -3 \\
\hline 19 & 10 & 10 & $\mathrm{~F}$ & 33 & 33 & 35 & 35 & 0 & 0 \\
\hline 20 & 6 & 12 & $\mathrm{~F}$ & 33 & 36 & 20 & 10 & 3 & -10 \\
\hline 21 & 5 & 9 & $M$ & 31 & 20 & 20 & 12 & -11 & -8 \\
\hline 22 & 3 & 20 & $\mathrm{~F}$ & 23 & 18 & 16 & 10 & -5 & -6 \\
\hline 23 & 6 & 20 & $M$ & 30 & 0 & 23 & 0 & -30 & -23 \\
\hline 24 & 7 & 15 & $\mathrm{M}$ & 31 & 25 & 20 & 16 & -6 & -4 \\
\hline 25 & 1 & 16 & $\mathrm{~F}$ & 20 & 8 & 12 & 6 & -12 & -6 \\
\hline 26 & 8 & 19 & $\mathrm{M}$ & 20 & 15 & 30 & 20 & -5 & -10 \\
\hline 27 & 5 & 17 & $M$ & 18 & 14 & 20 & 15 & -4 & -5 \\
\hline 28 & 8 & 4 & $M$ & 28 & 20 & 25 & 18 & -8 & -7 \\
\hline 29 & 8 & 15 & $\mathrm{~F}$ & 29 & 25 & 25 & 20 & -4 & -5 \\
\hline 30 & 7 & 16 & $M$ & 29 & 16 & 20 & 15 & -13 & -5 \\
\hline 31 & 3 & 18 & $\mathrm{~F}$ & 7 & 18 & 3 & 18 & 11 & 15 \\
\hline 32 & 3 & 17 & $\mathrm{~F}$ & 16 & 12 & 13 & 7 & -4 & -6 \\
\hline 33 & 5 & 16 & $\mathrm{~F}$ & 13 & 13 & 10 & 8 & 0 & -2 \\
\hline 34 & 7 & 20 & $\mathrm{~F}$ & 18 & 11 & 11 & 6 & -7 & -5 \\
\hline 35 & 7 & 18 & $\mathrm{M}$ & 27 & 22 & 15 & 11 & -5 & -4 \\
\hline 36 & 3 & 18 & $\mathrm{~F}$ & 18 & 20 & 9 & 12 & 2 & 3 \\
\hline 37 & 6 & 20 & $\mathrm{~F}$ & 28 & 22 & 40 & 30 & -6 & -10 \\
\hline 38 & 9 & 20 & $M$ & 32 & 32 & 35 & 30 & 0 & -5 \\
\hline 39 & 9 & 6 & $\mathrm{~F}$ & 34 & 30 & 25 & 13 & -4 & -12 \\
\hline
\end{tabular}


Tabella 6 Gruppo standard.

\begin{tabular}{|c|c|c|c|c|c|c|c|c|c|}
\hline Paz & Fagerström & Q-MAT & Sesso & $\mathrm{CO} 1^{\circ} \mathrm{coll}$. & $\mathrm{CO} 2^{\circ}$ coll. & $\begin{array}{l}\text { N. sigarette } \\
1^{\circ} \text { colloquio }\end{array}$ & $\begin{array}{l}\text { N. sigarette } \\
2^{\circ} \text { colloquio }\end{array}$ & $\begin{array}{c}\text { Variazione } \\
\text { CO }\end{array}$ & $\begin{array}{l}\text { Variazione } \\
\text { N. sigarette }\end{array}$ \\
\hline 1 & 5 & 18 & $\mathrm{M}$ & 23 & 34 & 10 & 15 & 11 & 5 \\
\hline 2 & 5 & 19 & $\mathrm{M}$ & 27 & 20 & 15 & 10 & -7 & -5 \\
\hline 3 & 3 & 16 & $\mathrm{~F}$ & 3 & 15 & 3 & 7 & 12 & 4 \\
\hline 4 & 6 & 12 & $\mathrm{~F}$ & 12 & 8 & 13 & 13 & -4 & 0 \\
\hline 5 & 8 & 20 & $M$ & 23 & 20 & 33 & 19 & -3 & -14 \\
\hline 6 & 5 & 18 & $\mathrm{~F}$ & 9 & 14 & 18 & 15 & 5 & -3 \\
\hline 7 & 6 & 12 & $\mathrm{~F}$ & 24 & 18 & 15 & 13 & -6 & -2 \\
\hline 8 & 6 & 17 & $\mathrm{M}$ & 28 & 28 & 20 & 12 & 0 & -8 \\
\hline 9 & 4 & 9 & $\mathrm{M}$ & 31 & 31 & 20 & 14 & 0 & -6 \\
\hline 10 & 7 & 18 & $\mathrm{M}$ & 31 & 23 & 30 & 15 & -8 & -15 \\
\hline 11 & 7 & 10 & $\mathrm{~F}$ & 27 & 27 & 30 & 30 & 0 & 0 \\
\hline 12 & 6 & 17 & $\mathrm{M}$ & 24 & 14 & 13 & 10 & -10 & -3 \\
\hline 13 & 4 & 12 & $\mathrm{M}$ & 21 & 10 & 15 & 12 & -11 & -3 \\
\hline 14 & 6 & 14 & $\mathrm{~F}$ & 16 & 15 & 20 & 13 & -1 & -7 \\
\hline 15 & 6 & 18 & $\mathrm{M}$ & 29 & 30 & 20 & 13 & 1 & -7 \\
\hline 16 & 7 & 18 & $\mathrm{M}$ & 31 & 24 & 40 & 40 & -7 & 0 \\
\hline 17 & 7 & 16 & $\mathrm{~F}$ & 34 & 29 & 20 & 13 & -5 & -7 \\
\hline 18 & 6 & 16 & $\mathrm{~F}$ & 11 & 7 & 16 & 7 & -4 & -9 \\
\hline 19 & 9 & 20 & $\mathrm{~F}$ & 26 & 35 & 37 & 27 & 9 & -10 \\
\hline 20 & 7 & 16 & $\mathrm{M}$ & 11 & 23 & 27 & 15 & 12 & -12 \\
\hline 21 & 5 & 20 & $\mathrm{~F}$ & 7 & 15 & 18 & 15 & 8 & -3 \\
\hline 22 & 8 & 8 & $\mathrm{M}$ & 14 & 28 & 30 & 15 & 14 & -15 \\
\hline 23 & 7 & 13 & $\mathrm{~F}$ & 12 & 23 & 20 & 20 & 11 & 0 \\
\hline 24 & 10 & 19 & $\mathrm{M}$ & 29 & 29 & 50 & 23 & 0 & -27 \\
\hline 25 & 9 & 14 & $\mathrm{~F}$ & 15 & 15 & 40 & 40 & 0 & 0 \\
\hline 26 & 7 & 9 & $\mathrm{~F}$ & 21 & 14 & 20 & 15 & -7 & -5 \\
\hline 27 & 1 & 16 & $\mathrm{~F}$ & 9 & 1 & 15 & 1 & -8 & -14 \\
\hline 28 & 10 & 18 & $\mathrm{M}$ & 25 & 15 & 45 & 20 & -10 & -25 \\
\hline 29 & 9 & 8 & $\mathrm{~F}$ & 19 & 7 & 27 & 20 & -12 & -7 \\
\hline 30 & 3 & 15 & $\mathrm{~F}$ & 11 & 11 & 5 & 6 & 0 & 1 \\
\hline 31 & 6 & 17 & $\mathrm{M}$ & 15 & 38 & 20 & 20 & 23 & 0 \\
\hline 32 & 6 & 17 & $\mathrm{M}$ & 10 & 18 & 20 & 20 & 8 & 0 \\
\hline 33 & 9 & 18 & $\mathrm{M}$ & 10 & 12 & 35 & 32 & 2 & -3 \\
\hline 34 & 9 & 3 & $\mathrm{M}$ & 14 & 18 & 20 & 20 & 4 & 0 \\
\hline 35 & 3 & 10 & $\mathrm{M}$ & 7 & 7 & 22 & 18 & 0 & -6 \\
\hline 36 & 4 & 16 & $\mathrm{~F}$ & 11 & 11 & 23 & 15 & 0 & -8 \\
\hline 37 & 8 & 17 & $\mathrm{~F}$ & 16 & 16 & 20 & 10 & 0 & -10 \\
\hline 38 & 8 & 20 & $\mathrm{M}$ & 35 & 22 & 22 & 20 & -13 & -12 \\
\hline 39 & 6 & 17 & $M$ & 4 & 7 & 25 & 22 & 3 & -3 \\
\hline
\end{tabular}




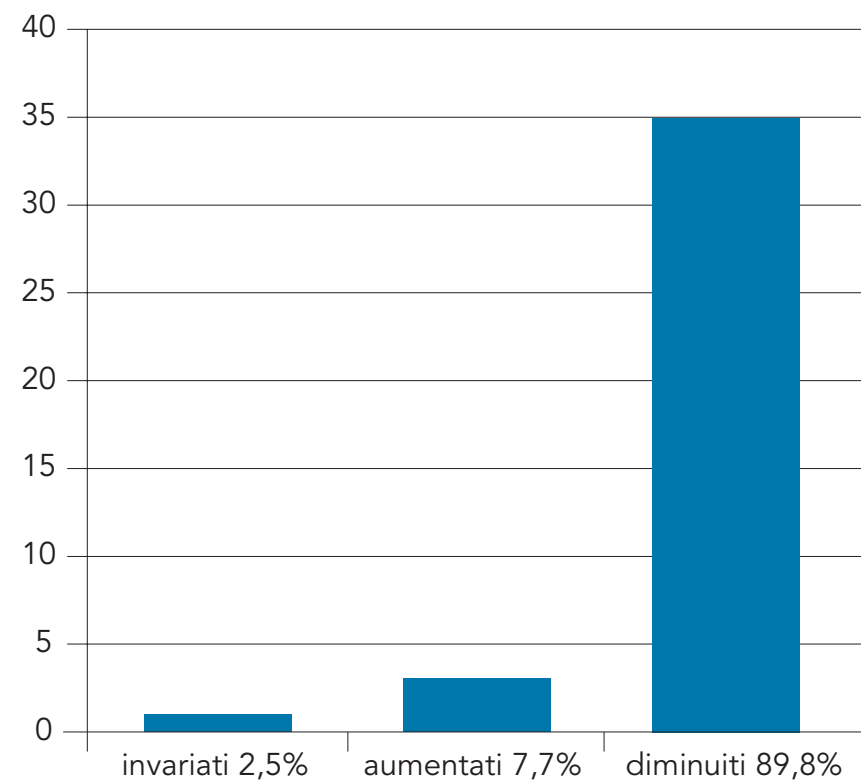

Figura 3A Variazione nel numero di sigarette del gruppo decisionale.

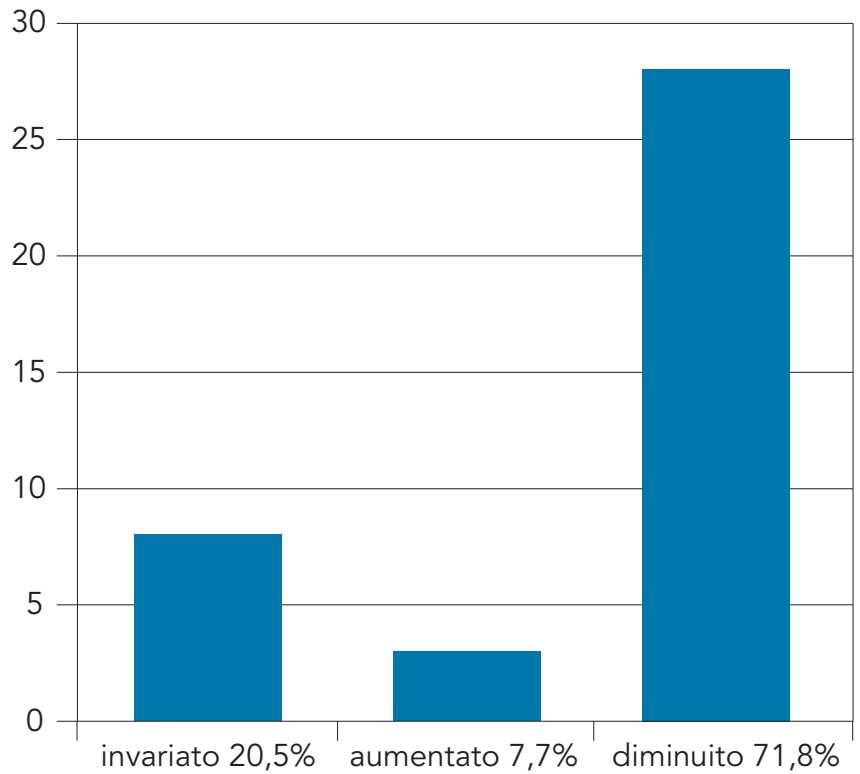

Figura 3B Variazione nel numero di sigarette del gruppo standard. gruppo decisionale rispetto a quello standard (77\% versus $41 \%)$. Se si valuta il numero di pazienti che presentano un aumento del livello di CO alla seconda visita, si osserva che è decisamente inferiore nel gruppo decisionale rispetto a quello standard (15\% versus $36 \%$ ). Infine, il numero di tabagisti che hanno mantenuto invariato il livello di CO tra prima e seconda misurazione è stato di 3 su 39 (8\%) nel gruppo decisionale, rispetto a 9 su 39 (23\%) nel gruppo standard. Per escludere che tale distribuzione fosse dovuta al caso, si è deciso di applicare il test t di Student alla riduzione media del $\mathrm{CO}$, rilevato tra prima e seconda visita, nei due gruppi. Si è così potuto osservare che la differenza fra le medie osservate è significativa e non casuale per $p<0,01 ; t=3,2501$; gradi di libertà $=76 ; p$ (livello di significatività): 0,0017. È stato utilizzato il foglio di calcolo Microsoft Excel, i cui risultati sono riportati in Tabella 7.

Abbiamo successivamente integrato il dato oggettivo della diminuzione del CO con il self-report relativo al numero di sigarette fuma- te: le Figure 3A e 3B mostrano le variazioni nei due gruppi. A una prima analisi si rileva, nel gruppo decisionale, una riduzione del numero di sigarette $(89,8 \%$ versus $71,8 \%)$. Va però precisato che, applicando il test statistico $\mathrm{t}$ di Student alle variazioni medie del numero di sigarette, tra prima e seconda visita, nei due gruppi, le differenze non sono risultate significative a meno del 5\% (Tabella 8).

In considerazione dei dati riportati in Tabella 2 e della omogeneità del campione, si può supporre che le differenze osservate tra i due gruppi non risentano né della gravità della dipendenza, valutata tramite test di Fagerström, né del livello di motivazione (punteggi Q-MAT).

\section{Conclusioni}

Il risultato di questo studio osservazionale mette in evidenza la possibilità di incidere sull'uso di tabacco, agendo fin dal primo colloquio in modo mirato attraverso il modello degli "snodi decisionali".

I dati sembrano infatti sostenere I'importanza della consapevolezza e della percezione della agency nel contrastare la dipendenza da tabacco, a prescindere dalla sua gravità e dal livello di motivazione. Trattandosi solo del primo colloquio, la minor aspirazione dalle sigarette, evidenziata dalla riduzione del $\mathrm{CO}$, rappresenta la fase iniziale del processo di cambiamento legato all'aumento della consapevolezza. Come esplicitato dal modello, ciò non si traduce necessariamente in una immediata riduzione del numero delle sigarette. II processo di cambiamento potrà poi essere consolidato ed esteso con incontri successivi.

Questo tipo di approccio ha ricadute positive, non solo sulle condizioni cliniche del paziente, ma anche sulla percezione della propria autoefficacia utile nel proseguimento del lavoro terapeutico, finalizzato alla cessazione tabagica. L'intervento descritto, infatti, ha l'obiettivo di apportare qualche stimolo già dal primo, e talora unico incontro, ma non è mosso dalla convinzione che tutto si possa risolvere in una sola volta [20].

L'efficacia del primo colloquio, momento in cui si presuppone che l'aspettativa e la motivazione siano massime, acquisisce ulteriore impor- 
Tabella 7 Test t di Student applicato alla riduzione media del CO nei due gruppi.

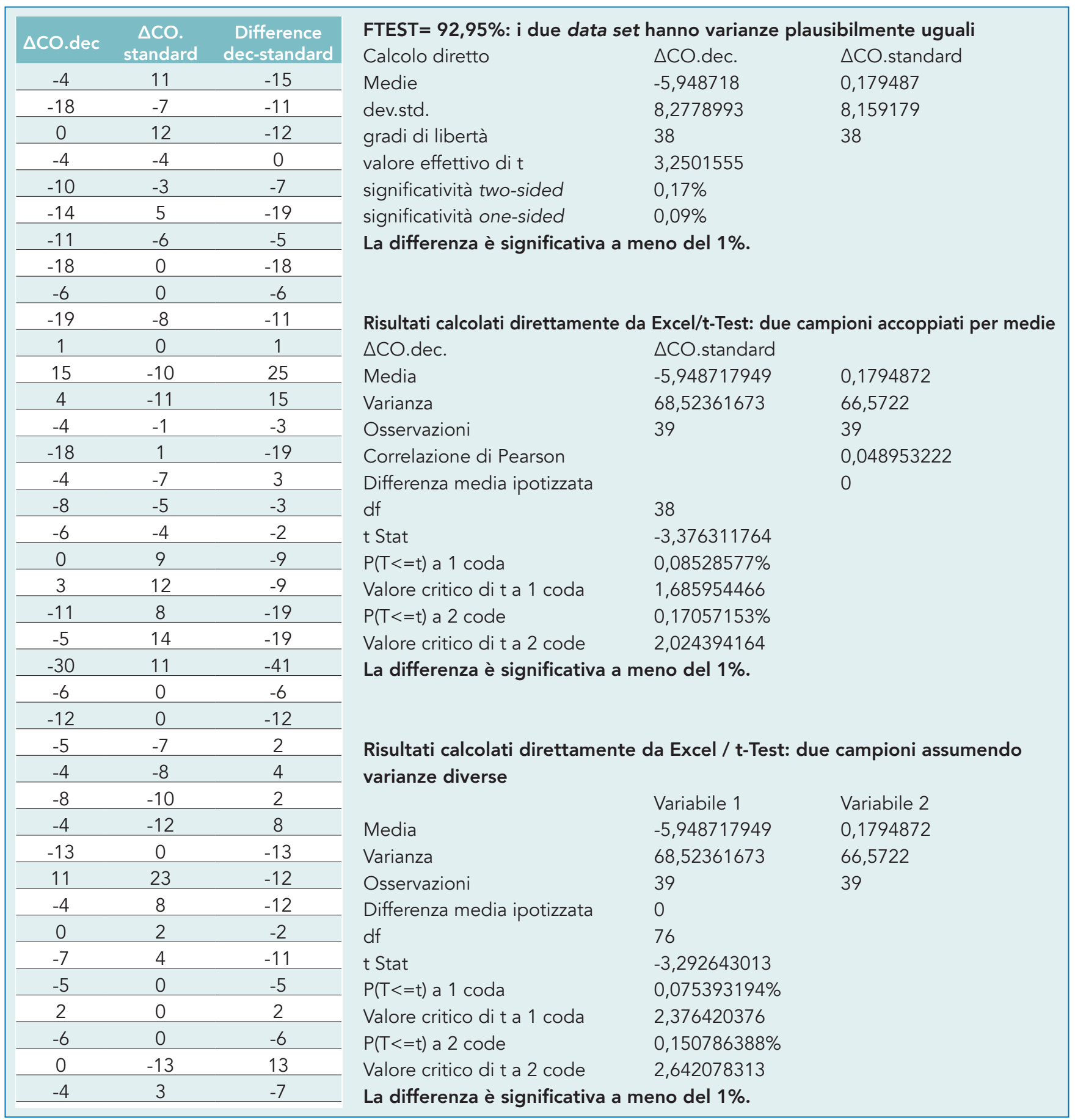

tanza se si tiene conto dei dropout. Anche in caso di interruzione precoce del percorso, la consapevolezza della propria agency può attivare una nuova modalità di percezione di sé, che il paziente ha la possibilità di applicare successivamente e non solo al consumo di tabacco.
D'altro canto, è certamente opportuno che questa acquisizione iniziale sia sostenuta, ampliata e rinforzata con il proseguimento del trattamento, come indicato dalle linee guida ENSP [35].

È ipotizzabile che rinforzare il ruolo centrale del paziente nel processo di cambiamento possa tra l'altro migliorare la compliance, aumentando l'aderenza al percorso ambulatoriale. Si è infatti osservato che questo tipo di approccio permette di ridurre il divario tra il terapeuta e il paziente.

Il lavoro sugli "snodi decisionali" fornisce al paziente informazioni che gli permettono di percepire la 
Tabella 8 Test t di Student applicato alle variazioni medie del numero di sigarette nei due gruppi.

\begin{tabular}{|c|c|c|c|c|c|}
\hline$\Delta$ sig.dec. & $\begin{array}{c}\Delta \text { sig. } \\
\text { standard }\end{array}$ & $\begin{array}{l}\text { Difference } \\
\text { dec-standard }\end{array}$ & \multirow{5}{*}{\multicolumn{3}{|c|}{ FTEST $=11,87 \%$ : i due data set hanno varianze significativamente diverse }} \\
\hline-5 & 5 & -10 & & & \\
\hline-38 & -5 & -33 & & & \\
\hline-10 & 4 & -14 & & & \\
\hline-8 & 0 & -8 & & & \\
\hline-4 & -14 & 10 & \multirow{7}{*}{$\begin{array}{l}\text { Calcolo diretto: } \\
\text { Medie } \\
\text { dev.std. } \\
\text { gradi di libertà } \\
\text { valore effettivo di t } \\
\text { significatività two-sided } \\
\text { significatività one-sided }\end{array}$} & dec & std \\
\hline-18 & -3 & -15 & & $-8,17949$ & $-6,1282051$ \\
\hline-12 & -2 & -10 & & 9,005471 & 6,97053757 \\
\hline-8 & -8 & 0 & & 38 & 38 \\
\hline-10 & -6 & -4 & & 1,110374 & \\
\hline-33 & -15 & -18 & & $27,04 \%$ & \\
\hline-5 & 0 & -5 & & $13,52 \%$ & \\
\hline-3 & -3 & 0 & \multirow{4}{*}{\multicolumn{3}{|c|}{$\begin{array}{l}\text { La differenza NON è significativa a meno del } 5 \% \text {. } \\
\text { Per esserlo, le ultime due percentuali dovrebbero essere inferiori al } 5 \% \text {. }\end{array}$}} \\
\hline 2 & -3 & 5 & & & \\
\hline-15 & -7 & -8 & & & \\
\hline-10 & -7 & -3 & & & \\
\hline-18 & 0 & -18 & \multirow{2}{*}{\multicolumn{2}{|c|}{ Usando le funzioni di Excel: }} & \\
\hline-6 & & 1 & & & \\
\hline-3 & -9 & 6 & \multirow{3}{*}{$\begin{array}{l}\text { Gradi di libertà } \\
\text { Test t a } 2 \text { code } \\
\text { Soglia di probabilità }\end{array}$} & 76 & \\
\hline 0 & -10 & 10 & & $26,44 \%$ & \\
\hline-10 & -12 & 2 & & $5,00 \%$ & \\
\hline-8 & -3 & -5 & \multirow{3}{*}{\multicolumn{3}{|c|}{$\begin{array}{l}\text { La differenza dei due data set è statisticamente significativa a meno } \\
\text { di questa soglia? NO }\end{array}$}} \\
\hline-6 & -15 & 9 & & & \\
\hline-23 & 0 & -23 & & & \\
\hline-4 & -27 & 23 & \multirow{4}{*}{\multicolumn{3}{|c|}{$\begin{array}{l}\text { Probabilità che la differenza non sia significativa: } 26,44 \% \\
\text { Valore critico di t: } 1,991673 \text {. Valore effettivo di t: } 1,124363\end{array}$}} \\
\hline-6 & 0 & -6 & & & \\
\hline-10 & -5 & -5 & & & \\
\hline-5 & -14 & 9 & & & \\
\hline-7 & -25 & 18 & \multirow{2}{*}{$\begin{array}{l}\text { Test t a } 1 \text { coda } \\
\text { Soglia di probabilità }\end{array}$} & $13,22 \%$ & \\
\hline-5 & -7 & 2 & & $5,00 \%$ & \\
\hline-5 & 1 & -6 & \multirow{3}{*}{\multicolumn{3}{|c|}{$\begin{array}{l}\text { La differenza dei due data set è statisticamente significativa a meno } \\
\text { di questa soglia? NO }\end{array}$}} \\
\hline 15 & 0 & 15 & & & \\
\hline-6 & 0 & -6 & & & \\
\hline-2 & -3 & 1 & \multirow{2}{*}{\multicolumn{3}{|c|}{$\begin{array}{l}\text { Probabilità che la differenza non sia significativa: } 21,07 \% \\
\text { Valore critico di t: }-1,66515 \text {. Valore effettivo di t: }-1,12436\end{array}$}} \\
\hline-5 & 0 & -5 & & & \\
\hline-4 & -6 & 2 & \\
\hline 3 & -8 & 11 & & & \\
\hline-10 & -10 & 0 & & & \\
\hline-5 & -12 & 7 & & & \\
\hline-12 & -3 & -9 & & & \\
\hline
\end{tabular}

possibilità di prendere delle decisioni e di attuare in modo attivo un cambiamento.

In questo nuovo contesto, il medico non fornisce indicazioni da seguire, ma sostiene il vero protagonista del percorso di cure.

[Tabaccologia 2021; XIX(1):21-32] https://doi.org/10.53127/tblg-2021-A006
Franco Badii, Sarah Grossi

Ser.D. - Dipartimento Salute Mentale e Dipendenze ASL2, Sistema Sanitario Regione Liguria

\section{Corresponding author:}

Franco Badii

Ser.D. - Dipartimento Salute Mentale

e Dipendenze ASL2

Sistema Sanitario Regione Liguria via S. Lucia 11/1 - 17100 Savona (SV) Tel. 0198405350

f.badii@asl2.liguria.it

Disclosure: gli autori dichiarano I'assenza di conflitto d'interessi.

Fondi e finanziamento: nessuno. 


\section{Bibliografia}

1. Robinson TE, Berridge KC. The neural basis of drug craving: an incentive-sensitization theory of addiction. Brain Res Rev 1993;18:247-91. https://doi.org/10.1016/0165-0173(93)90013-P

2. Berridge KC, Robinson TE. What is the role of dopamine in reward: hedonic impact, reward learning, or incentive salience? Brain Res Rev 1998;28:309-69. https://doi. org/10.1016/S0165-0173(98)00019-8

3. Robinson TE, Berridge KC. The psychology and neurobiology of addiction: an incentive-sensitization view. Addiction 2000;95: S91-117. https://doi.org/10.1080/09652140050111681

4. Robinson TE, Berridge KC. Incentive-sensitization and addiction. Addiction 2001;96:103-14. https://doi.org/10.1046/ j.1360-0443.2001.9611038.x

5. Berridge KC. The debate over dopamine's role in reward: the case of incentive salience. Psychopharmacology (Berl) 2007;191:391-431. https://doi.org/10.1007/s00213-006-0578-x

6. Robinson TE, Berridge KC. The incentive sensitization theory of addiction: some current issues. Philos Trans R Soc Lond B Biol Sci 2008;363:3137-46. https://doi.org/10.1098/ rstb.2008.0093

7. Berridge KC, Robinson TE. Liking, wanting, and the incentive-sensitization theory of addiction. Am Psychol 2016; 71:670-9. https://doi.org/10.1037/amp0000059

8. Godini L, Lelli L, Campone B, Ciampi E, Corsi E, Ramella Car-

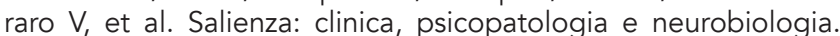
Riv Psich 2015;50:255-69. https://doi.org/10.1708/2098.22680

9. Robinson TE, Berridge KC. Addiction. Ann Rev Psychol 2003;54:2553. https://doi.org/10.1146/annurev.psych.54.101601.145237

10. Badii F. Un modello fisiopatologico e clinico della addiction. Dal fare al dire 2013;suppl 3:26-33.

11. Berne, E. Analisi transazionale e psicoterapia. Un sistema di psichiatria sociale e individuale. Edizioni Astrolabio Roma, 1961.

12. Woollams S, Brown M. Analisi transazionale. Psicoterapia della persona e delle relazioni. Edizioni Cittadella Assisi, 1985.

13. Di Clemente CC. Addiction and change. How addictions develop and addicted people recover. Guildford Press New York, 2006.

14. Lewis M. Addiction and the brain: development non disease. Neuroethics 2017;10:7-18. https://doi.org/10.1007/ s12152-016-9293-4

15. Dill B, Holton R. The addict in us all. Front Psychiatry 2014;5:139. https://doi.org/10.3389/fpsyt.2014.00139

16. Holton R, Berridge K. Compulsion and choice in addiction in Heater N, Segal G (a cura di) Addiction and choice. Rethinking the relationship. University Press Oxford, 2017.

17. Nakken C. Personalità Viziose. Comprendere il processo di dipendenza e riprendere il controllo di sé. Tecniche Nuove Milano, 1999.

18. McCambridge J, Strang J. The efficacy of single session motivational interviewing in reducing drug consumption and perceptions of drug related risk and harm among young people: results from a multisite cluster randomized trial. Addiction 2002;99:3952. https://doi.org/10.1111/j.1360-0443.2004.00564.x

19. Berman AH, Forsberg L, Dubreej N, Kallmen H, Hermansson $U$. Single-session motivational interviewing for drug detoxification inpatients: effects on self-efficacy, stages of change and substance use. Subst Use Misuse 2010;45:348-402. https://doi.org/10.3109/10826080903452488

20. Cannistrà F, Piccirilli F. Terapia a seduta singola. Principi e pratiche. Giunti Editore Firenze, 2018.

21. Miller W, Rollnick S. Il colloquio motivazionale. Aiutare le persone a cambiare. Edizioni Centro Studi Erickson Trento, 1991.

22. Rosengren D.B. Guida pratica al counseling motivazionale. Manuale per professionisti sociali e sanitari. Edizioni Centro Studi Erickson Trento, 2011.

23. Tinghino B. Stili di vita e tabagismo, strumenti di counselling per motivare al cambiamento. II Pensiero Scientifico Editore Roma, 2012.

24. Badii F. II counseling decisionale per il trattamento della dipendenza. Dal Fare al Dire 2005; 1:29-39.

25. American Psychiatric Association (APA). Diagnostic and statistical manual of mental disorders. American Psychiatric Publishing Washington, 2013.

26. Fagerström KO, Schneider NG. Measuring nicotine dependence: a review of the Fagerström tolerance questionnaire. J Behav Med 1989;12:159-82. https://doi.org/10.1007/ BF00846549

27. Heatherton TF, Koziowski LT, Frecker RC, Fagerström KO. The Fagerström Test for nicotine dependence: a revision of the Fagerström Tolerance Questionnaire. Brit J Addict 1991; 86:111927. https://doi.org/10.1111/j.1360-0443.1991.tb01879.x

28. Pomerleau CS, Majchrzak MI, Pomerleau OF. Nicotine dependence and the Fagerström Tolerance Questionnaire: a brief review. J Substance Abuse 1989;1:471-7. https://doi. org/10.1016/S0899-3289(20)30011-0

29. Aubin HJ, Lagrue G, Legeron P, Azoulaï G, Pélisolo S, Humbert R, et al. Questionnaire de motivation à l'arrêt du tabac (Q-MAT). Alcool Addictol 2004;26:311-6.

30. Susumu S, Nishimura K, Koyama H, Tsukino M, Oga T, Hajiro $\mathrm{T}$, et al. Optimal cutoff level of breath carbon monoxide for assessing smoking status in patients with asthma and COPD, Chest 2003;24:1749-54. https://doi.org/10.1378/chest.124.5.1749

31. Javors MA, Hatch JP, Lamb RJ. Cut-off levels for breath carbon monoxide as a marker for cigarette smoking. Addiction 2005;100:159-67. https://doi.org/10.1111/j.1360-0443. 2004.00957.x

32. Bize R, Burnand B, Mueller Y, Rege-Walther M, Camain JY, CornuzJ. Biomedical risk assessment as an aid for smoking cessation. Cochrane Database Syst Rev 2012;12:CD004705. https://doi.org/10.1002/14651858.CD004705.pub4

33. Wald NJ, Idle M, Boreham J, Bailey A. Carbon monoxide in breath in relation to smoking and carboxyhemoglobin levels. Thorax 1981;36:366-9.

34. Middleton ET, Morice AH. Breath carbon monoxide as an indication of smoking habit. Chest 2000;117:758-63. https://doi.org/10.1378/chest.117.3.758

35. European Network for Smoking and Tobacco Prevention aisbl (ENSP), Società Italiana di Tabaccologia (SITAB), Istituto di ricerche farmacologiche "Mario Negri" IRCCS. Linee guida per la dipendenza da tabacco. European Network for Smoking and Tobacco Prevention aisbl (ENSP) Brussels, 2020. 